POS $\quad$ PROCEEDINGS

\title{
Test, Integration and Commissioning of Monitored Drift Tube Chambers for the ATLAS Barrel Muon Spectrometer
}

Jörg Dubbert*

Max-Planck-Insitut für Physik

Munich, Germany

E-mail: joerg.dubbert@mppmu.mpg.de

Manfred Groh

Max-Planck-Insitut für Physik

E-mail: manfred.groh@mppmu.mpg.de

\section{Sandra Horvat}

Max-Planck-Insitut für Physik

E-mail: sandra.horvat@mppmu.mpg.de

\section{Oliver Kortner}

Max-Planck-Insitut für Physik

E-mail: Oliver.Kortner@mppmu.mpg.de

\section{Hubert Kroha}

Max-Planck-Insitut für Physik

E-mail: hubert.kroha@mppmu.mpg.de

\section{Susanne Mohrdieck-Moeck}

Max-Planck-Insitut für Physik

E-mail: susanne.mohrdieck@mppmu.mpg.de

\section{Robert Richter}

Max-Planck-Insitut für Physik

E-mail: robert.richter@mppmu.mpg.de

\section{Otmar Biebel}

Department für Physik, Ludwig-Maximilians-Universität München

E-mail: otmar.biebel@physik.uni-muenchen.de

\section{Johannes Elmsheuser}

Department für Physik, Ludwig-Maximilians-Universität München

E-mail: johannes.elmsheuser@physik.uni-muenchen.de

\section{Marion Erlebach}

Department für Physik, Ludwig-Maximilians-Universität München

E-mail: marion.erlebach@physik.uni-muenchen.de

\section{Frank Fiedler}


Department für Physik, Ludwig-Maximilians-Universität München E-mail: frank.fiedler@physik.uni-muenchen.de

\section{Doris Merkl}

Department für Physik, Ludwig-Maximilians-Universität München

E-mail: doris.merkl@physik.uni-muenchen.de

\section{Ralf Hertenberger}

Department für Physik, Ludwig-Maximilians-Universität München E-mail: ralf.hertenberger@physik.uni-muenchen.de

\section{Thomas Nunnemann}

Department für Physik, Ludwig-Maximilians-Universität München E-mail: thomas.nunnemann@physik.uni-muenchen.de

\section{Raphael Mameghani}

Department für Physik, Ludwig-Maximilians-Universität München E-mail: raphael.mameghani@physik.uni-muenchen.de

\section{Felix Rauscher}

Department für Physik, Ludwig-Maximilians-Universität München E-mail: felix.rauscher@physik.uni-muenchen.de

\section{Dorothee Schaile}

Department für Physik, Ludwig-Maximilians-Universität München E-mail: dorothee.schaile@physik.uni-muenchen.de

\section{Arnold Staude}

Department für Physik, Ludwig-Maximilians-Universität München E-mail: arnold.staude@physik.uni-muenchen.de

\section{Raimund Ströhmer}

Department für Physik, Ludwig-Maximilians-Universität München E-mail: raimund.stroehmer@physik.uni-muenchen.de

\section{Cornelius F. Vollmer}

Department für Physik, Ludwig-Maximilians-Universität München E-mail: fritz.vollmer@physik.uni-muenchen.de 
The ATLAS experiment at the Large Hadron Collider (LHC) at CERN is currently being assembled and to be ready for data taking in 2007. In the barrel region of its muon spectrometer, a toroidal air-core magnet is instrumented with three layers of Monitored Drift Tube (MDT) chambers as precision tracking detectors; the middle and outer layers of drift chambers also carry Resistive Plate chambers (RPCs) of the trigger system. The barrel part of the spectrometer consists of a total of 682 muon stations, with sizes ranging from $1 \mathrm{~m}^{2}$ to $11 \mathrm{~m}^{2}$. The installation of the muon detectors has started in January 2005. At the Max-Planck-Institut für Physik and the Ludwig-Maximilians-University in Munich, 88 MDTs of the second largest chamber type-each covering an area of up to $8 \mathrm{~m}^{2}$ - are being built for the outermost barrel region. The MDT chambers have to pass a series of stringent tests before installation to ensure their proper operation in the experiment. At the production site in Munich, these tests include gas tightness, high voltage stability and measurements of the noise rate, and the response to cosmic muons. In addition, the individual wire positions and electronic time offsets of the drift tubes are determined from the cosmic ray data. After shipment to CERN, a subset of the tests is repeated and the MDT chambers are integrated on a common support frame with the trigger chambers. The completed muon station is is further checked with cosmic rays. All results are stored in the ATLAS commissioning database and constitute an important basis for LHC data taking. We present the test methods and results, an overview on the integration work of the muon detectors and report on the experience with the installation of the first stations in the ATLAS experiment.

International Europhysics Conference on High Energy Physics

July 21st - 27th 2005

Lisboa, Portugal

*Speaker. 


\section{Introduction}

The ATLAS (A Toroidal LHC ApparatuS) experiment is one of the two multi-purpose detectors at the Large Hadron Collider (LHC) at CERN. Its muon spectrometer [1] has been designed to be capable of stand-alone operation (i.e. it does not require information from the inner detector or calorimeters to reconstruct muon tracks) with a resolution of better than $10 \%$ for muon momenta up to $1 \mathrm{TeV}$.

In the barrel region of the muon spectrometer a three point sagitta measurement with Monitored Drift Tube (MDT) chambers is used to determine the muon momentum. To achieve the desired precision, each MDT must reach a resolution of better than $50 \mu \mathrm{m}$.

ATLAS MDT chambers [1] consist of two multilayers, each built of 3 or 4 layers of densely packed drift tubes mounted on a support frame; the chamber sizes vary from about $1 \mathrm{~m}^{2}$ to $11 \mathrm{~m}^{2}$ while tube lengths range from 1 to $6 \mathrm{~m}$. The individual drift tubes have an outer diameter of 30 $\mathrm{mm}$ and a wall thickness of $400 \mu \mathrm{m}$. A single anode wire with a diameter of $50 \mu \mathrm{m}$ is stretched in the center of the tube and fixed only at the tube ends; the sag of the wires in the tubes can be compensated by bending the chamber with respect to the support frame.

The determination of the impact radius of a charged particle within a drift tube is based on the measurement of the drift time of the primary ionization electrons to the anode wire; a socalled rt-relation is then used to convert drift time information to a radius prediction. In the case of MDT chambers this rt-relation can be determined by an iterative self-calibrating algorithm, the auto-calibration [2]. An average single tube resolution of $80 \mu \mathrm{m}$ has been achieved with an $\mathrm{Ar} / \mathrm{CO}_{2}=93 / 7$ gas mixture at 3 bar absolute pressure at a gas gain of $2 \times 10^{4}$. The maximum drift time for electrons from hits near the tube wall to the anode wire is about $700 \mathrm{~ns}$.

The geometry of the drift tube chambers, as well as the positions of all MDTs in the detector, are constantly monitored by optical alignment systems $[1,3]$.

\section{Tests and Commissioning of MDT Chambers}

The Max-Planck-Institut für Physik (MPI) and the Ludwig-Maximilians-University (LMU) at Munich built 88 MDT chambers for the outermost barrel layer of the muon spectrometer, most of them consisting of 432 drift tubes arranged in $2 \times 3$ layers and measuring $4 \mathrm{~m} \times 2.2 \mathrm{~m} \times 0.5 \mathrm{~m}$.

To ensure the necessary geometrical precision and the proper operation of the MDT chambers, the assembly process is monitored at each step [4,5]. The most important parameters of the individual drift tubes - gas leak rate, anode wire tension, dark current and the wire position, the latter with a precision of a few $\mu \mathrm{m}$ with an X-Ray tomograph-are measured immediately after their production. During chamber construction geometrical parameters-such as e.g. the position of reference surfaces at both tube ends and the position of the chamber support frame during glueing of the tube layers - are measured. Combined with the knowledge of the single tube anode wire positions, these measurements yield the chamber geometry, i.e. the position of all anode wires and the layer parameters of the MDT.

After the chamber has been assembled, each connection of a drift tube to the common gas distribution bar is checked to conform with the leak rate limit of $1 \cdot 10^{-8} \mathrm{mbar} 1 / \mathrm{s}$ and the leak rate of each multilayer is determined from the temperature corrected pressure drop over a time span of 

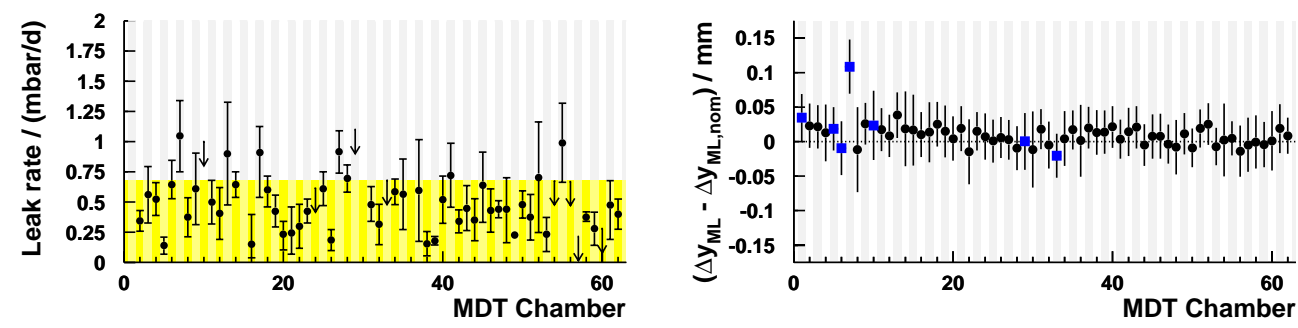

Figure 1: Results for 71 MDT chambers from tests and measurements at the production site. Chambers are ordered by their date of production, ranging from 2001 to 2005. Left: Leak rate of MDT chambers. The shaded area is the allowed leak rate. Right: Measurement of the displacement of the two multilayers from their nominal vertical separation at the read-out chamber side. The blue square points mark repaired chambers. The measurement error for individual wire positions is $25 \mu \mathrm{m}$. The error bars are the RMS spread of the deviations of the individual drift tubes from the multilayer fit.

several days. Almost all chambers fulfill the stringent leak rate limit, the maximum measured leak rate is 1.5 times the specification (see fig. 1 left).

The MDT chamber is equipped with the front-end electronics and subjected to a high voltage test. A noise test is performed with a random trigger signal both without and with high voltage to be able to differentiate external noise caused by bad connections or the active front-end electronics of each tube or internal discharges inside the gas volume.

The chamber is then installed in the Cosmic Ray Test Stand [6,7] where its response to cosmic muons is measured and analyzed. The data taken at the Cosmic Ray Stand also allows the determination of the individual anode wire positions of a MDT chamber with an accuracy of $\mathscr{O}(10 \mu \mathrm{m})$. From these results, the chamber geometry-(multi)layer distances, tilt angles and the mean wire distances - can be reconstructed. The analysis methods and the setup of the Cosmic Ray Test Stand are described in $[8,9]$. All 71 MDTs tested so far show and homogeneous response across the chamber and a consistent geometry (compare fig. 1 right).

\section{Integration of MDT Chambers at CERN}

Upon their arrival at CERN, the MDT chambers are again subjected to a test programme (leak, high voltage and noise tests), to discover any damage which might have occurred during transport and to verify their proper operation. The MDTs are then integrated with their respective trigger chambers and the completed muon station-MDT, trigger chamber and Level 1 trigger electronics-is commissioned with cosmic rays. The requirements to be fulfilled by the MDT chambers are defined in [10].

After the MDT chamber has passed all tests, it is equipped with B-field and alignment sensors and alignment extension plates, as well as survey targets on its outer side which are used for positioning of the muon station during and after installation in the ATLAS detector.

Due to their position on the outside of the ATLAS toroid magnet coils, the MDT chambers built at Munich require a special common support frame to carry their RPC trigger chamber. The completed muon station is rotated in a special frame to the angle at which it will be installed in the ATLAS detector and the height of the MDT chamber with respect to the bearings of the common 
support frame is adjusted with an accuracy of $1 \mathrm{~mm}$. In the rotator, the MDT chamber is also bend to follow the wire sag by $\pm 74 \mu \mathrm{m}$ or $\pm 179 \mu \mathrm{m}$, depending on the installation angle. The precision and repeatability of the sag adjustment is better than $10 \mu \mathrm{m}$.

After integration, the muon station is placed in a simple cosmic ray test stand for a complete system test. For the MDT, the drift time spectra and pulse height distributions for each tube are recorded and the chamber hit profile is checked. Similar tests ensure the proper operation of the RPC trigger chamber and the Level 1 trigger electronics.

\section{Conclusions}

The Max-Planck-Institut für Physik and the Ludwig-Maximilians-University Munich have built 88 MDT chambers for the outermost barrel region of the ATLAS muon spectrometer. At the Cosmic Ray Facility at Munich, 71 of these chambers have been tested and calibrated after assembly. All chambers show an uniform response and a consistent geometry, well within the allowed limits. In the early installation phase of the ATLAS muon spectrometer at the beginning of 2005, the first four of these muon stations were successfully integrated with their trigger chambers, commissioned at CERN and installed in the detector. The integration of the remaining 84 chambers is forseen until the end of the year. The installation will resume early in 2006.

\section{References}

[1] ATLAS Muon Collaboration, ATLAS Muon Spectrometer Technical Design Report, CERN/LHCC/97-22, CERN (1997)

[2] M. Deile, Optimization and Calibration of the Drift-Tube Chambers for the ATLAS Muon Spectrometer, $\mathrm{PhD}$ thesis, Ludwig-Maximilians-Universität Munich (2000)

[3] H. van der Graaf et al., RasNiK, an Alignment System for the ATLAS MDT Barrel Muon Chambers Technical System Description, NIKHEF/ET38110 (2000)

[4] F. Bauer et al., Construction and test of MDT chambers for the ATLAS muon spectrometer, Nucl.Instrum.Meth. A461 (2001) 17-20

[5] S. Horvat, Study of the Higgs Discovery Potential in the Process $p p \rightarrow H \rightarrow 4 \mu, \mathrm{PhD}$ thesis, Zagreb University and Max-Planck-Institut für Physik Munich (2005)

[6] O. Kortner, Schauerproduktion durch hochenergetische Myonen und Aufbau eines Höhenstrahlungsprïfstands für hochauflösende ATLAS-Myonkammern, $\mathrm{PhD}$ thesis, Ludwig-Maximilians-Universität Munich (2002)

[7] O. Biebel et al., A Cosmic Ray Measurement Facility for ATLAS Muon Chambers, e-Print physics/0307147 (2003)

[8] O. Biebel et al., Test and Calibration of Large Drift Tube Chambers with Cosmic Rays, to be published in IEEE Trans.Nucl.Sci.

[9] F. Rauscher, Untersuchung des Verhaltens von Driftrohren bei starken Gamma-Bestrahlung sowie Vermessung von Driftrohrkammern mit Hilfe von Myonen der kosmischen Höhenstrahlung, $\mathrm{PhD}$ thesis, Ludwig-Maximilians-Universität Munich (2005)

[10] The Commissioning Working Group, MDT Commissioning Procedures Guidelines for Certifying RFI Chambers, ATL-COM-MUON-2004-022, CERN (2004) 